2005

\title{
Spatiotemporal Networks In Addressable Excitable Media
}

\author{
Mark Tinsley \\ Jianxia Cui
}

Florin V. Chirila

Annette Taylor

Shi Zhong

See next page for additional authors

Follow this and additional works at: https://researchrepository.wvu.edu/faculty_publications

\section{Digital Commons Citation}

Tinsley, Mark; Cui, Jianxia; Chirila, Florin V.; Taylor, Annette; Zhong, Shi; and Showalter, Kenneth, "Spatiotemporal Networks In Addressable Excitable Media" (2005). Faculty Scholarship. 681.

https://researchrepository.wvu.edu/faculty_publications/681 
Authors

Mark Tinsley, Jianxia Cui, Florin V. Chirila, Annette Taylor, Shi Zhong, and Kenneth Showalter 


\title{
Spatiotemporal Networks in Addressable Excitable Media
}

\author{
Mark Tinsley, ${ }^{1}$ Jianxia Cui, ${ }^{1}$ Florin V. Chirila, ${ }^{1}$ Annette Taylor, ${ }^{2}$ Shi Zhong, ${ }^{1}$ and Kenneth Showalter ${ }^{1}$ \\ ${ }^{1}$ Department of Chemistry, West Virginia University, Morgantown, West Virginia 26506-6045, USA \\ ${ }^{2}$ Department of Chemistry, University of Leeds, Leeds LS29JT, United Kingdom
}

(Received 17 February 2005; published 15 July 2005)

\begin{abstract}
Spatiotemporal networks are studied in a photosensitive Belousov-Zhabotinsky medium that allows both local and nonlocal transmission of excitation. Local transmission occurs via propagating excitation waves, while nonlocal transmission takes place by nondiffusive jumps to destination sites linked to excited sites in the medium. Static, dynamic, and domain link networks are experimentally and computationally characterized. Transitions to synchronized behavior are exhibited with increasing link density, and powerlaw relations are observed for first-coverage time as a function of link probability.
\end{abstract}

\section{DOI: 10.1103/PhysRevLett.95.038306}

Networks offer a framework for understanding connectivities arising from interactions such as those between computers on the Internet, neurons in an organism, or elements in a power grid [1-4]. Recent theoretical studies of small world and scale-free networks have provided insights into many natural and man-made systems with complex connectivity $[3,4]$. We report here on a study of dynamic networks in an experimental system, the photosensitive Belousov-Zhabotinsky (BZ) reaction [5,6]. Experimental and computational studies are carried out on three levels of systems: static link, dynamic link, and domain link networks. Using addressable excitable media [7-9], we model local nearest-neighbor interactions by the spread of reaction-diffusion waves, while nonlocal excitations are introduced by nondiffusive shortcuts defined in the system. The shortcuts remain fixed throughout the experiment in the static link network but continually change in the dynamic link network. We report on the transition to synchronous behavior with an increase in link density, and we characterize power-law relations between first-coverage time and link probability. Our study follows recent investigations of synchronization in networks of linked dynamical systems, including coupled oscillatory systems [10-13]. It is also relevant to recent studies of dynamical networks in biological systems, including models of neuronal tissues [3,14-16] and the susceptible-infected-recovery-susceptible (SIRS) description of disease spreading [17-19].

Our simplest system is the static link model, where the nonlocal connections are described by fixed nondiffusive jumps. The experimental system consists of the photosensitive BZ reaction in which the trisbipyridine-Ru(II) catalyst is immobilized in a thin layer of gel that is bathed in continuously refreshed catalyst-free solution. A video projector and video camera, interfaced with a computer, allow real-time feedback for perturbing the light sensitive medium and monitoring its response. The quasi-twodimensional gel is partitioned into an array of cells via the imposed illumination image, and each cell is assigned a probability of being connected to another cell randomly chosen in the array. After the links are assigned [20], the
PACS numbers: $82.40 . \mathrm{Bj}, 05.45 . \mathrm{Xt}, 05.65 .+\mathrm{b}, 89.75 . \mathrm{Hc}$

experiment is started by initiating a wave at the center of the medium, which propagates outward through the cells. Upon reaching a preset threshold coverage in a cell, arbitrarily defined as the excitation of $50 \%$ of the grid points in the cell, the initiation of a wave is triggered at a linked destination cell by increasing its excitability.

The spatiotemporal behavior of the static link model is shown in Fig. 1, where 1(a) and 1(b) reveal how the initial wave gives rise to new wave initiations due to nondiffusive
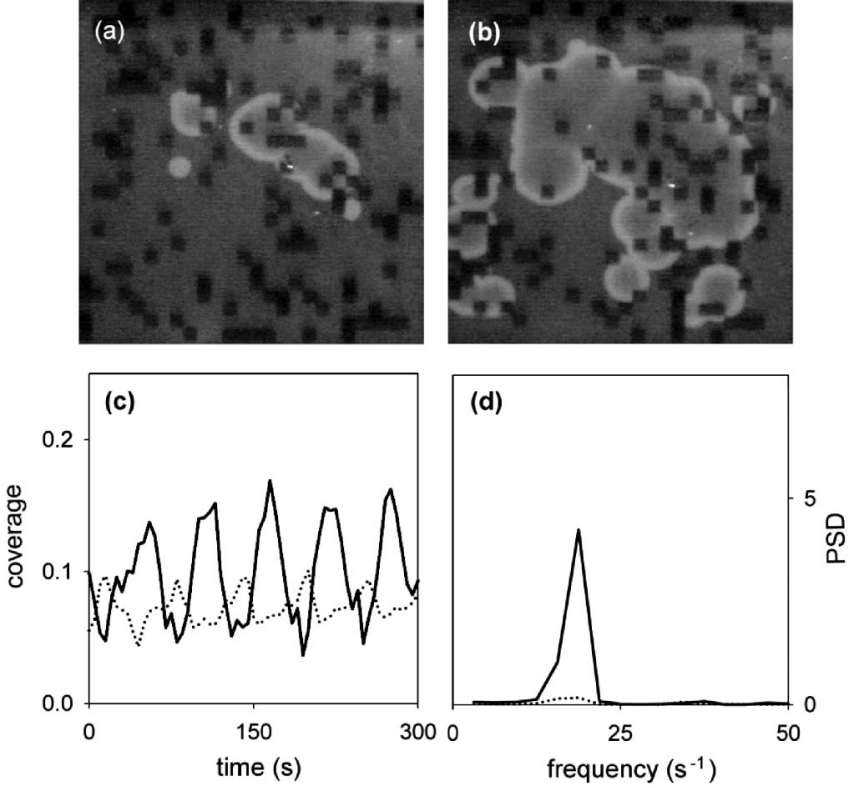

FIG. 1. Spatiotemporal behavior in the static link model arising from diffusive and nondiffusive wave transmission. (a),(b) Two snapshots with a $15 \mathrm{~s}$ interval show excitation evolution for 300 links in a network of $26 \times 26$ square cells, with each cell composed of $10 \times 10$ grid points. (c) Time series show fractional excitation coverage for 300 (solid line) and 20 (dotted line) links; (d) power spectral density corresponding to the time series in (c). The link probability $p=0.44$ and 0.03 , respectively, is defined as the average number of links per cell. Waves were initiated at destination cells by reducing the light intensity at those cells, shown by the black squares. 
jumps and how these waves subsequently give rise to further new initiations. We measure the asymptotic spatiotemporal behavior by the fractional coverage, which is the number of excited grid points divided by the total number of grid points in the system. Figure 1(c) shows time series of the fractional coverage for experiments with 20 and 300 links, and the corresponding power spectra are shown in Fig. 1(d). An apparent increase in periodicity with an increase in the number of shortcuts is exhibited, where periodicity in the fractional coverage is indicative of spatiotemporal synchronization of the system.

A less rigid system is the dynamic link model, in which the shortcuts are determined dynamically according to a probability $p$ of each cell having a nondiffusive connection. Hence, when a cell reaches a threshold coverage, there is a probability $p$ that it will trigger the initiation of a new wave at another cell randomly selected in the network. Figures 2(a) and 2(b) show the spatiotemporal behavior of the dynamic link model, where waves are continually initiated at random locations corresponding to links from excited cells. Figure 2(c) shows the fractional coverage time series for two different jump probabilities, $p=0.01$ and 0.50, and Fig. 2(d) shows the corresponding power spectra. We see there is little correlation in the coverage as a function of time at low jump probability; however, the coverage becomes periodic at higher probability, indicating spatiotemporal synchronization of the system.
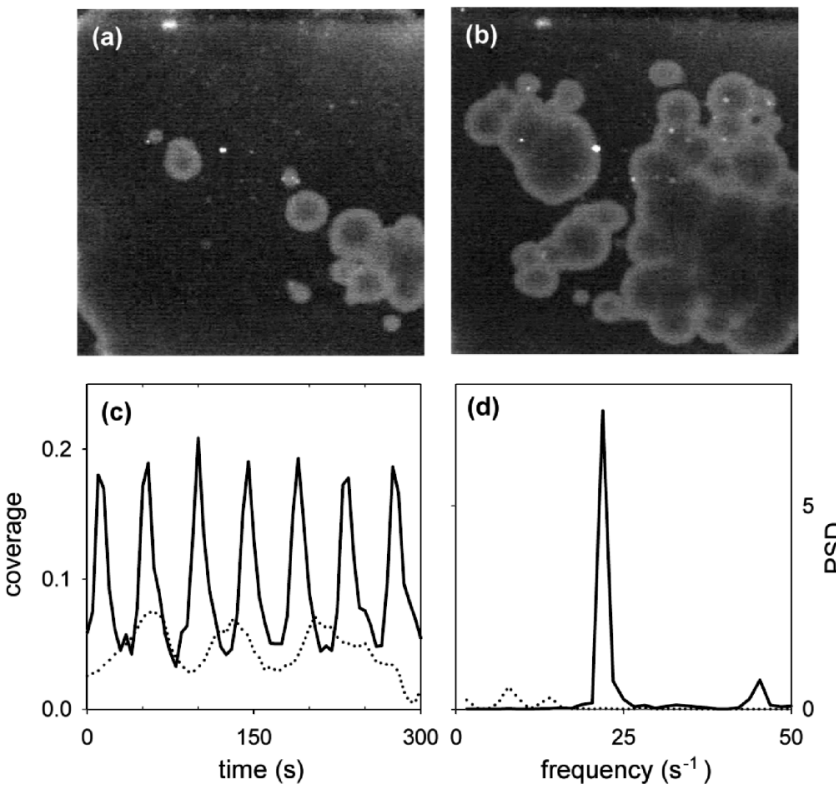

FIG. 2. Spatiotemporal behavior in the dynamic link model. (a),(b) Two snapshots with a $15 \mathrm{~s}$ interval show excitation evolution for a link probability $p=0.5$. (c) Time series show fractional excitation coverage for $p=0.01$ (dotted line) and $p=0.5$ (solid line); (d) power spectral density corresponding to the time series in (c). Methods and conditions are the same as described in Fig. 1; initiation sites were removed from images for clarity.
In the static link model, shortcuts are established only between certain cells, and wave initiations always occur at the same link destinations. The dynamic link model relaxes the connectivity, with all cells having a certain probability of repeatedly establishing links to other cells at different random locations within the network. We note, however, that nonlocal connectivity may occur between populations of cells rather than to random locations [21], and we have therefore refined the dynamic link model to give the $d o$ main link model. The number of cells per domain is determined according to a power-law distribution [22], and the shortcuts are established between the clusters of cells. Figure 3(a) shows a collection of domains in our simulations [24] using the Oregonator model for the photosensitive BZ reaction [25-27], where the local reactiondiffusion behavior within a domain is initiated by nondiffusive jumps from other domains. Links are established as in the dynamic link model, with the exception that the destination of a nondiffusive jump may not occur to the domain of the jump origin. Since each cell has the probability $p$ of establishing a link to another domain, the rate of
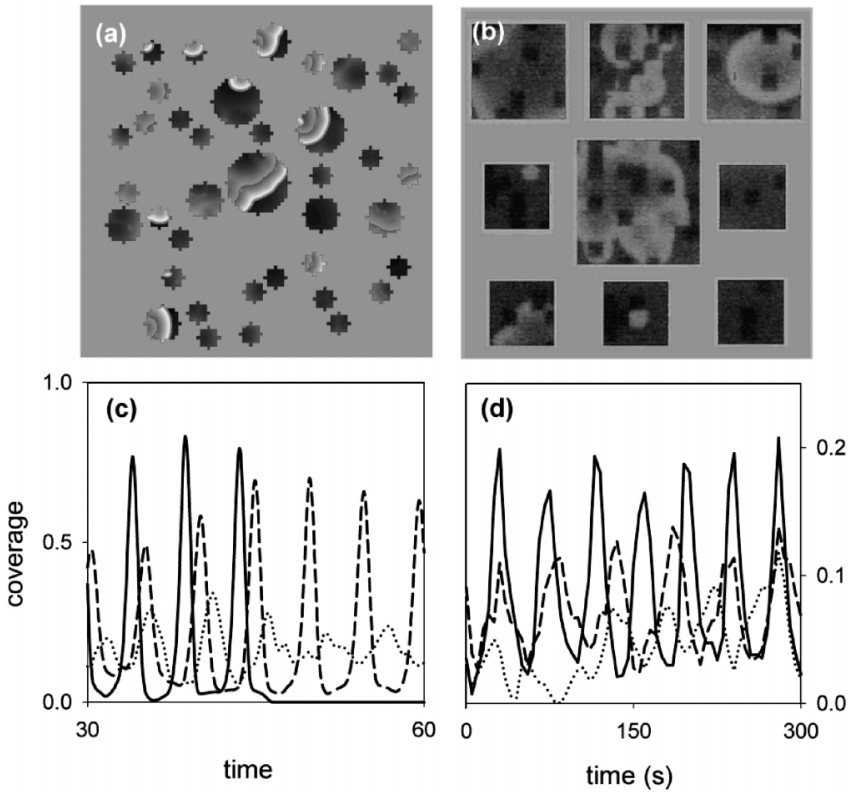

FIG. 3. Spatiotemporal behavior in the domain link model. (a) Excitation distribution in simulations with $p=0.5$, where cells of $10 \times 10$ grid points are partitioned into 40 domains composed of 260, 190, 100, and 50 cells. (b) Excitation distribution in experimental system with $p=0.7$. Isolated squares of catalyst-loaded gel are partitioned into arrays of cells of $10 \times 10$ grid points to define nine domains composed of 81, 49, and 36 cells. (c) Time series show fractional excitation coverage for simulated behavior, with $p=0.1$ (dotted line), 0.3 (dashed line), 0.5 (solid line), and (d) experimental behavior, with $p=0.05$ (dotted line), 0.40 (dashed line), 0.70 (solid line). Experimental methods and conditions are the same as described in Fig. 1. Numerical simulations were carried out using the Oregonator model for the photosensitive BZ reaction [24]. 
nondiffusive jumps from a particular domain is proportional to the size of that domain. The rate of nondiffusive jumps to a particular domain is also proportional to the size of the domain in the limit of many domains and a large overall cell population. Figure 3(c) shows time series for three different link probabilities in the domain model. We see little periodicity in fractional coverage at low probability, $p=0.1$, while at an intermediate probability, $p=$ 0.3 , significant periodicity is apparent. Periodic oscillations are displayed at high probability, $p=0.5$, in which all domains are locked in almost complete synchronization. The most striking feature of this time series is the complete collapse of activity at $t \approx 46.6$, where all cells in all domains return to the excitable stationary state. A timelapse sequence of this collapse reveals that the medium is not completely synchronized immediately before the transition; however, the remaining active domain was unable to initiate new wave activity because all other domains were in the refractory state. The time of collapse was not predictable in subsequent simulations; however, the likelihood of collapse increased with increasing probability $p$.

An experimental implementation of the domain model is depicted in Fig. 3(b), where clusters of cells forming domains are imposed on physically separated BZ gels [28]. We see a similar spreading of excitation as in the dynamic link model, except now nondiffusive jumps are required for spreading from one domain to another. In Fig. 3(d), three time series are shown corresponding to jump probabilities $p=0.05,0.40$, and 0.70 . At low jump probability, the domains are excited in an uncorrelated manner, and the time series for the fractional coverage displays little periodicity. At high jump probability, however, the excitation occurs synchronously throughout the domains, as shown by the periodic time series. The collapse of activity observed in the simulations was not found in our limited number of experiments, possibly due to the effects of unavoidable experimental inhomogeneities in excitability. The longer wave initiation times observed in the experiments (discussed below) may also play a role in the absence of collapse.

Another important measure of the spread of excitation is the first-coverage time, defined as the time necessary for the medium at each grid point to be excited at least once. The first-coverage time measures the growth of the network during the initial transient period before the asymptotic behavior is established. Figure 4 shows experimental measurements of first-coverage time as a function of link probability for both the static and the dynamic link models. We see a power-law relation for each model, $\tau_{\text {static }}=$ $0.45 p^{-0.27}$ and $\tau_{\text {dynamic }}=0.39 p^{-0.27}$, with very similar slopes. Also shown in Fig. 4 are computational results for the first-coverage time as a function of link probability in the static and dynamic link models, where we again see power-law relationships, $\tau_{\text {static }}=0.34 p^{-0.26}$ and $\tau_{\text {dynamic }}=0.36 p^{-0.25}$, again with very similar slopes. The

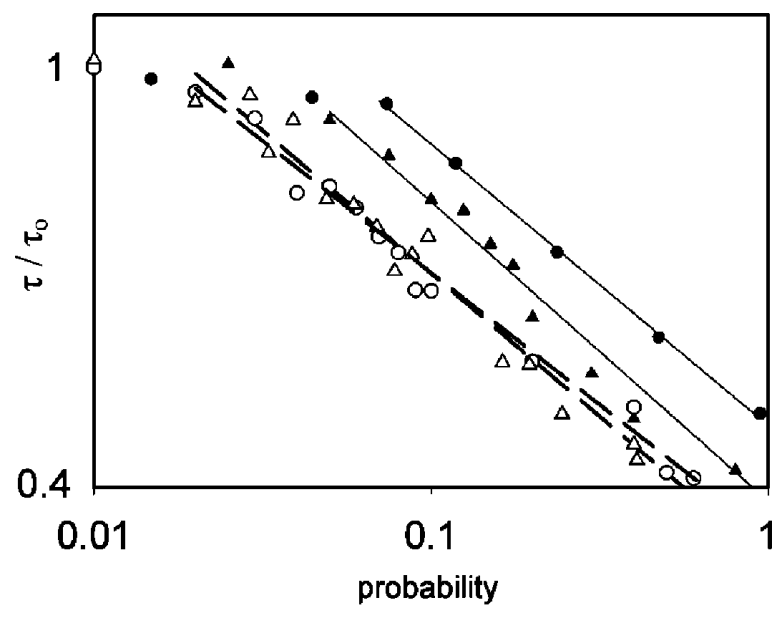

FIG. 4. Log-log plot of first-coverage time $\tau$ as a function of link probability $p$. Each value of $\tau$ represents the average of six experimental measurements for the static link (filled circles) and the dynamic link (filled triangles) models. Values of $\tau$ as a function of $p$ obtained from simulations [24] represent an average of 10 values computed for the static link (open circles) and dynamic link (open triangles) models. Value of $\tau_{o}$ is coverage time for a single wave; values of $\tau$ at the lowest probabilities were omitted in obtaining the linear regression lines. Methods and conditions were the same as described in Figs. 1-3

power laws arise from positive feedback in the growth of the network, where the rate of new wave initiations increases with each expanding excitation. In both the experiments and calculations, there is a plateau in the firstcoverage time at low jump probability, which is close to the normalized time for a single propagating wave to cover the medium. This plateau corresponds to negligible feedback in the network growth.

We have presented highly idealized models of dynamical networks with diffusive and nondiffusive excitations. Additional features can be incorporated into these models that correspond to the complexities of realistic settings. Varying excitabilities, for example, can be introduced by imposing illumination heterogeneity, which can be controlled pixel by pixel. We have carried out simulations with heterogeneous excitabilities, where each grid point is assigned an illumination intensity that is chosen randomly from a Gaussian distribution. While not exhaustive, our studies showed quantitative but no qualitative changes from the behavior described above for weak to moderate heterogeneity. However, spontaneous wave initiations occur with high heterogeneity, and the spread of excitation is then no longer determined by nondiffusive jumps. The initiation time is another important element in determining the spatiotemporal dynamics. The wave initiation time was significantly faster in the simulations than in the experiments (based on inherent system time scales such as the refractory period). This difference was found to be caused by residual light intensity during wave initiations in the 
experiments. The shorter simulated initiation times resulted in smaller first-coverage times, although the slope of the $\log \tau$ vs $\log p$ plot was little affected. The agreement between the experimental and simulated first-coverage times was improved by introducing an initiation delay time in the simulations ( 0.80 for both the static and the dynamic models), which shifted the log-log plots to the positions shown in Fig. 4.

Spatiotemporal networks are relevant to a variety of systems with diffusive and nondiffusive connectivity, especially within and among biological organisms $[3,14-$ $18,21]$. Such networks also present new challenges for network theory $[10,11]$. We note that even the static link model has a distinctly dynamical character, since the fixed nondiffusive jumps are triggered by propagating waves. The dynamic and domain link models have an additional stochastic character, with new links established according to a conditional probability of initiating new excitations at link destination cells [29]. The remarkable similarity between the first-coverage time power laws for the static and the dynamic link models points to an underlying commonality in the growth dynamics of these systems.

We thank the National Science Foundation (CHE0415392), the Office of Naval Research, and the W.M. Keck Foundation for supporting this research. K. S. thanks the Alexander von Humboldt Foundation for supporting research visits to the Fritz-Haber-Institut der Max-PlanckGesellschaft.

[1] D. J. Watts and S. H. Strogatz, Nature (London) 393, 440 (1998).

[2] R. Albert, H. Jeong, and A.-L. Barabasi, Nature (London) 401, 130 (1999).

[3] S. H. Strogatz, Nature (London) 410, 268 (2001).

[4] A.-L. Barabasi and R. Albert, Rev. Mod. Phys. 74, 47 (2002).

[5] A. N. Zaikin and A.M. Zhabotinsky, Nature (London) 225, 535 (1970).

[6] L. Kuhnert, Nature (London) 319, 393 (1986).

[7] J. Wolff, A. G. Papathanasiou, I. G. Kevrekidis, H. H. Rotermund, and G. Ertl, Science 294, 134 (2001).

[8] T. Sakurai, E. Mihaliuk, F. Chirila, and K. Showalter, Science 296, 2009 (2002).

[9] J. Wolff, A. G. Papathanasiou, H. H. Rotermund, G. Ertl, M. Katsoulakis, X. Li, and I. G. Kevrekidis, Phys. Rev. Lett. 90, 148301 (2003).

[10] M. Barahona and L. M. Pecora, Phys. Rev. Lett. 89, 054101 (2002).

[11] H. Hong, M. Y. Choi, and B. J. Kim, Phys. Rev. E 65, 026139 (2002).

[12] X. Wang and G. Chen, Int. J. Bifurcation Chaos Appl. Sci. Eng. 12, 187 (2002).
[13] P. Gade and C. Hu, Phys. Rev. E 62, 6409 (2000).

[14] A. Roxin, H. Riecke, and S. A. Solla, Phys. Rev. Lett. 92, 198101 (2004).

[15] T. I. Netoff, R. Clewley, S. Arno, T. Keck, and J. A. White, J. Neurosci. 24, 8075 (2004).

[16] L. F. Lago-Fernández, R. Huerta, F. Corbacho, and J. A. Sigüenza, Phys. Rev. Lett. 84, 2758 (2000).

[17] M. Kuperman and G. Abramson, Phys. Rev. Lett. 86, 2909 (2001).

[18] M. Girvan, D. S. Callaway, M. E. J. Newman, and S. H. Strogatz, Phys. Rev. E 65, 031915 (2002).

[19] M. E. J. Newman, Phys. Rev. E 66, 016128 (2002).

[20] A unidirectional static link is assigned by randomly choosing a source cell $i$ and a destination cell $j(j \neq i)$ from the $N$ cells of the network. For a static $n$-link network, this process is repeated $n$ times at the start of each experiment or simulation. The probability $p$ for the static network is defined as the average number of links per cell $p=n / N$.

[21] O. Sporns and J.D. Zwi, Neuroinformatics 2, 145 (2004).

[22] The domain sizes $s$ were determined using a Pareto-like power-law distribution [23], $n=6850 s^{-2.00}$ for the experiments and $n=89320 \mathrm{~s}^{-2.05}$ for the simulations, where $n$ is the number of domains containing $s$ cells. The multiplicative coefficients were chosen to yield convenient domain sizes for the experiments and simulations.

[23] R. Gunther, L. Levitin, B. Shapiro, and P. Wagner, Int. J. Theor. Phys. 35, 395 (1996).

[24] Numerical studies were carried out using an Oregonator model of the photosensitive BZ reaction [25-27]: $\frac{\partial u}{\partial t}=$ $D_{u} \nabla^{2} u+\frac{1}{\epsilon}\left[u-u^{2}-(\phi+f v) \frac{u-q}{u+q}\right], \frac{\partial v}{\partial t}=u-v$ where $u$ and $v$ are the dimensionless concentrations of $\mathrm{HBrO}_{2}$ and $\mathrm{Ru}(\mathrm{bpy})_{3}^{{ }^{+}}$, respectively, $D_{u}$ is the diffusion coefficient of $\mathrm{HBrO}_{2}, \nabla^{2}$ is the Laplacian operator, and $\phi$ represents the rate of bromide production from the irradiation. A wave was initiated at cells with $\phi=0$, while the surrounding medium was maintained in the excitable state with $\phi=2.8$. The Euler method was used for the numerical integration, with the parameters $f=1.4, q=0.0002$, $\epsilon=0.01, D_{u}=1.0, d x=0.15, d t=0.001$, where $\epsilon$ and $q$ are scaling parameters and $f$ is an adjustable stoichiometry parameter. Behavior very similar to that in Figs. 1 and 2 was obtained in simulations of the static and dynamic link models.

[25] R. J. Field and R. M. Noyes, J. Chem. Phys. 60, 1877 (1974).

[26] J. J. Tyson and P. C. Fife, J. Chem. Phys. 73, 2224 (1980).

[27] H. J. Krug, L. Pohlmann, and L. Kuhnert, J. Phys. Chem. 94, 4862 (1990).

[28] The domains were composed of collections of cells determined by the light imposed grid, where each domain was on a square section of gel physically separated from the other sections.

[29] D. R. Cox and H. D. Miller, The Theory of Stochastic Processes (Wiley, New York, 1965). 